\title{
Polyamines and Gut Microbiota
}

\author{
Rosanna Tofalo ${ }^{1 *}$, Simone Cocchi ${ }^{2}$ and Giovanna Suzzi ${ }^{1}$ \\ ${ }^{1}$ Faculty of Bioscience and Technology for Food, Agriculture and Environment, University of Teramo, Teramo, Italy, ${ }^{2}$ Farmacie \\ Comunali di Romano di Lombardia, Bergamo, Italy
}

Keywords: polyamines, gut microbiome, human health, probiotics, fermented foods

The microbiota of gut is the community of microbes living in an individual's gastrointestinal tract. Several bacterial genera and species act in a concerted manner to establish metabolic interactions with the host (1). Although there is a general high interest in the study of metabolite flow across the microbe-host, at present, only some studies are targeting specific metabolites produced by intestinal microbiota such as polyamines (PAs) (2).

\section{POLYAMINES AND GUT MICROBIOTA}

Polyamines can be defined as small polycationic molecules with a wide array of biological functions including gene regulation, stress resistance, cell proliferation and differentiation, and are associated to both eukaryotic and prokaryotic cells (3).

In human cells spermine, spermidine, and putrescine are the main PAs. Putrescine is produced in the cytoplasm of cells by decarboxylation of ornithine catalyzed by the enzyme ornithine decarboxylase (ODC). Spermine and spermidine are synthesized by S-adenosyl-methionine

OPEN ACCESS

Edited by:

Antonio F. Tiburcio,

University of Barcelona, Spain

Reviewed by:

Syed Sifraz Shah,

Forman Christian College, Pakistan

Rafael Peñafiel,

University of Murcia, Spain

*Correspondence:

Rosanna Tofalo

rtofalo@unite.it

Specialty section: This article was submitted to Nutrition and Food Science Technology,

a section of the journal

Frontiers in Nutrition

Received: 29 November 2018 Accepted: 01 February 2019

Published: 25 February 2019

Citation:

Tofalo R, Cocchi S and Suzzi G (2019) Polyamines and Gut Microbiota. Front. Nutr. 6:16 doi: 10.3389/fnut.2019.00016 decarboxylase (AdoMetDC) and a transferase enzyme, catalyzing the transfer of the aminopropyl group to the primary amine group of putrescine or spermidine, respectively (4). The ingested food is the major source of PAs in the lumen, and the upper parts of intestine adsorb the majority of these compounds for growth processes throughout the body (5). The gut microbiota is considered the main responsible of PAs level in the lower part of intestine (6). Polyamines in the colonic lumen are transferred into the bloodstream via the colonic mucosa (7). Intracellular PAs levels are regulated by endogenous biosynthesis, degradation and exogenous transport. Both endocytic and solute carrier-dependent mechanisms have been described for polyamine uptake in the gut lumen (8). In eukaryotic cells they are involved in several physiological functions since they are able to bind to several anionic macromolecules such as DNA, RNA, proteins, and acidic phospholipids (9). The PAs involvement in maintaining chromatin structure and membrane stability and regulating ion-channels and scavenging free radicals has also been reported (10), as well as their role as second messengers in protein and nucleic acid synthesis for normal cell division and growth (11). In particular, cellular PAs availability contributes to tissue homeostasis of the gastrointestinal mucosa, the rates of epithelial cell division and apoptosis, by modulating the expression of various growthrelated genes (12). In general, PAs are involved in several important cellular processes and their disregulation can affect growth, aging and several diseases such as cancer, neurodegeneration and metabolic disorders (13). To maintain good intracellular PAs contents, biosynthetic and catabolic processes are activated and highly regulated. For example, a high intracellular PAs levels are related with cell growth, whereas the inhibition of ODC decreases cellular PAs (12). On the contrary, its overexpression induces an increased level of PAs in human gut, a result that has been related with gastrointestinal cancers (14).

As regards bacteria, new putative phyla (134) other than the traditional ones (30) have been identified using culture-independent metagenomic sequencing and single-cell sequencing (15). However, the studies of PAs distribution in bacteria have been limited to culturable species and few bacterial species have been studied (16). 
The bacterial PAs include spermidine, homospermidine, norspermidine, putrescine, cadaverine, and 1,3-diaminopropane with putrescine and spermidine being the most common PA $(17,18)$. Furthermore, because of high bacterial diversity, some microorganisms produce sym-homospemidine rather than spermidine or produce only a diamine and some bacteria do not produce PAs, such as Staphylococcus aureus (19).

Different bacterial species, up to 1,000 , constitute the intestinal microbiota. This community of microorganisms (bacteria, archaea, fungi, protozoa, viruses) is responsible for the metabolism of non- digested food components and it can supply to the host nutrients such as amino acids and vitamins and other biologically active substances (20). In general, this microbial consortium is subject to fluctuations due to different factors such as environment, diet, disease states and many others (21). The microorganisms colonizing the gut can contribute to the overall health of the host or be pathogenic, invading the host, and causing diseases under certain conditions (22).

The majority of the studies on gut microbiota are focused on bacteria, even if all the biota plays important roles in host health and disease (23). The high-throughput sequencing techniques based on the amplification of the $16 \mathrm{~S}$ rRNA identified more than 120 different prokaryotic phyla with only 31 phyla included cultured species (24). Moreover, the majority of species that constitute the gut microbiota belong mainly to four phyla: Firmicutes, Bacteroidetes, Actinobacteria, Proteobacteria, and among these Firmicutes and Bacteroidetes are dominant phyla in agreement with the high-throughput sequences carried out the last 10 years. The dominant species belong to the families Bacillaceae, Enterobacteriaceae, Corynebacteriaceae, and Bacteroidaceae, with a prevalence of anaerobic species, uncultured yet (25). Culturomics is a new strategy that could improve the study of microorganisms of the human gut microbiota (26).

As regards Archaea, non-methanogenic and methanogenic species are present in human gut microbiota, with the latter producing methane during anaerobic fermentations. These species belong to Euryarchaeota phylum. A very small fungal community is present in the human gut with three major phyla: Ascomycota 63\%, Basidiomycota 32\%, and Zygomycota $3 \%$.

Putrescine, cadaverine, spermidine, and spermine are the main PAs encountered in bacteria (Figure 1). Their synthesis is highly regulated at molecular level through a concerted biosynthesis and uptake mechanisms, as well as by degradation and efflux processes. Their production relies on the presence of amino acidic precursor or other intermediates which are then converted into functional PAs (28). Besides de novo synthesis pathways, PAs uptake can be controlled through specific transport systems. They are highly conserved among bacteria. The best-known examples are two ABC transporters described in Escherichia coli that are specific for either putrescine or spermidine and two antiporters, exchanging putrescine for ornithine and lysine for cadaverine (29).

Recent studies highlighted the involvement of PAs in bacterial pathogenesis. A clear example is Shigella spp. an intracellular pathogen associated to enteric syndrome in humans (30). For instance, due to mutations and deletions, cadaverine is lost from Shigella spp., improving the pathogenicity process, because cadaverine has a protective effect on intestinal mucosa from enterotoxins. Spermidine accumulation increases Shigella resistance to oxidative stress and its survival in macrophages [for a review see (28)].

\section{CONTRIBUTION OF GUT MICROBIOTA TO PAs FORMATION}

Almost all foods contain PAs; they are abundant in soybeans, mushrooms, wheat germ, beef, pork, chicken livers, oranges, turban shell viscera, and green tea leaves. A great part of PAs introduced by foods is absorbed in the small intestine, whereas microbiota produce these compounds in great amounts in the large bowel (2). Little is known about the production and degradation of biogenic amines (BAs) by gut microbiota and in particular PAs. Recently, isolates from the human gut, belonging to many different species, were found to produce and degrade BAs at different levels depending on the strains (16). Putrescine and spermidine, important metabolites of intestinal bacteria, are present in the intestinal lumen in concentrations ranging from 0.5 to $1 \mathrm{mM}$ in healthy humans (6). Gut microorganisms can synthesize putrescine, spermine and spermidine, that are present at millimolar concentrations, and play a major role in providing PAs for the high demand of these compounds in intestine. Bacteria use PAs for cell to cell communication, cellular signals and cell differentiation and the bacterial metabolism of these compounds determines the PAs intestinal content. The main studies were performed in E. coli, even if it is a minor microbial component in the human intestine and its PAs biosynthetic pathway seems to be different from those present in dominant gut microbiota (31). Few studies report data on metabolites produced by intestinal microbiota and their functions, in particular short fatty acids (32) and PAs $(33,34)$. In addition, Bacteroides spp. and Fusobacterium spp. can synthesize putrescine and spermidine in vitro and in vivo (35). Recently Nakamura et al. (20) found that in colonic lumen putrescine is produced by different bacteria from collective biosynthetic pathways depending on a complex exchange of metabolites.

The environmental stimuli can modulate the gut microbiota metabolism as well as the absorption and release of PAs. Noack et al. (36) reported that indigestible polysaccharides pass into the large intestine and are fermented with the production of shortchain fatty acids and lower $\mathrm{pH}$, that can modify the intestinal microbiological metabolism and composition, and stimulate intestinal PAs content synthesis. In general, the fermentable carbohydrates present in the large bowel contribute to increase the bacterial PAs formation with consequent beneficial effects on the gut mucosa. In addition, by using in silico analysis, novel PAs biosynthetic and transport proteins have been found. There are few studies on PAs biosynthetic pathway carried out by dominant intestinal microorganisms. In fact, great part of gut bacteria utilizes carboxyspermidine dehydrogenase and carboxyspermidine decarboxylase (CASDC) for spermidine biosynthesis, whereas $E$. coli utilizes S-adenosylmethionine decarboxilase and spermidine synthase (8). The species in 


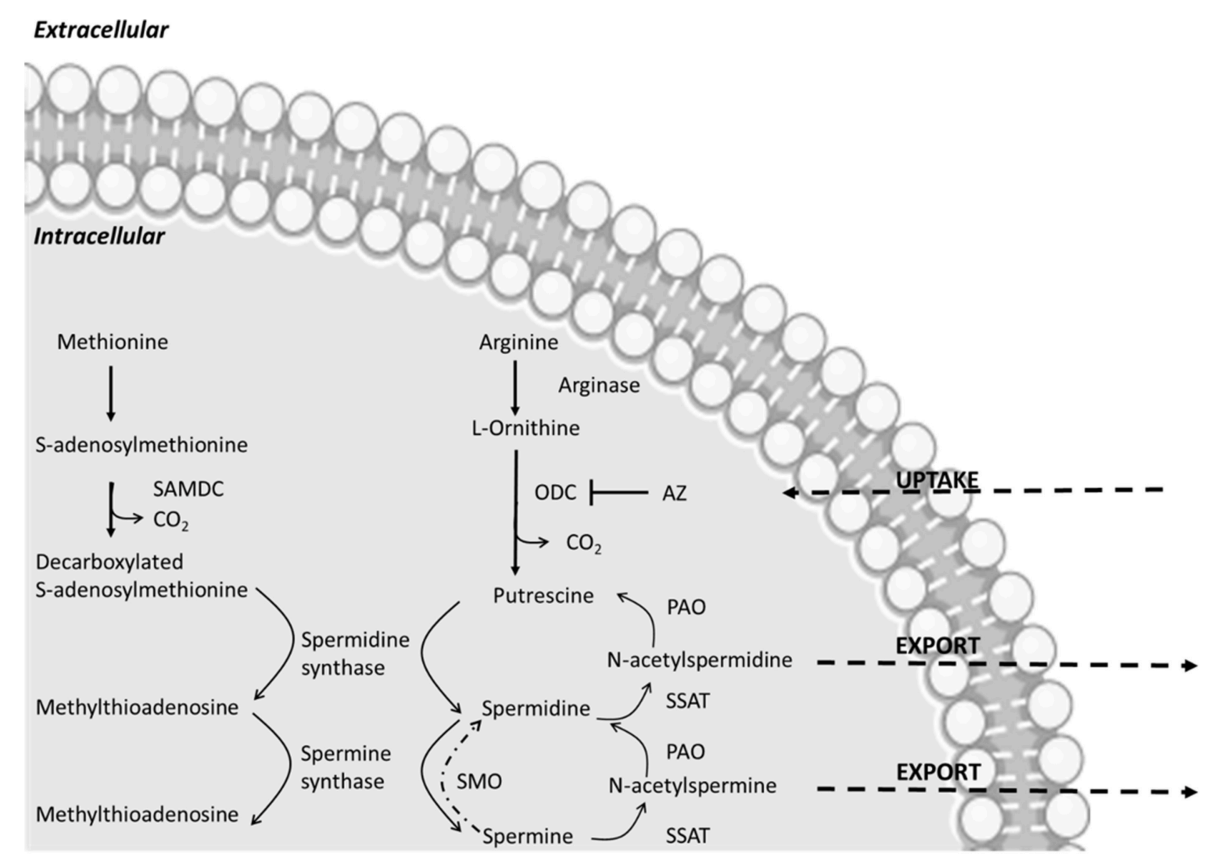

FIGURE 1 | Main polyamine metabolic pathways modified from Linsalata et al. (27). The abbreviations used are as follows: ODC, ornithine decarboxylase; SAMDC, S-adenosylmethionine decarboxylase; SSAT, spermidine-spermine-N-acetyl transferase; PAO, polyamine oxidase; AZ, antizyme; SMO, spermine oxidase. AZ inactivates ODC, increases polyamine efflux and decreases polyamine uptake.

the genus Bacteroides, which is predominant in intestine of humans with 20 of 56 most abundant species, harbors CASDC homologs (31) that are essential for spermidine biosynthesis contributing to the normal bacterial growth (37). Sugiyama et al. (8) evaluated the capacity of 32 bacterial species dominant in human gut to produce PAs in the cell and in supernatants, suggesting the presence of new genes and transporters. As many colonic microbial species do not possess complete synthetic pathways to produce PAs (38), it is possible to suppose the existence of metabolic interactions among bacterial species in the gut. Kitada et al. (39) showed that putrescine concentration produced by a mixed culture of different microbial species from gut microbiota was higher than that obtained with the single cultures. They demonstrated that a mixed culture of $E$. coli and Enterococcus faecalis produced the highest quantities of putrescine when the $\mathrm{pH}$ of the medium drops to neutral, suggesting the involvement of bacterial acid resistance system (40). A new pathway for putrescine formation was identified, from arginine to agmatine, with the cooperation of these two species. In presence of low $\mathrm{pH}$, the acid resistance system of E. coli produces agmatine from arginine, and an arginineagmatine antiporter exchanges extracellular arginine for the intracellular end product of decarboxylation, agmatine (41). Enterococcus faecalis, through an agmatine/putrescine antiporter, metabolizes the agmatine to putrescine by agmatine deiminase pathway, yielding ATP, $\mathrm{CO}_{2}$, and $\mathrm{NH}_{3}$ (42). The presence of other bacteria, such as Bifidobacterium spp. producing acid compounds in gut, favors the induction of this new pathway for putrescine production $(39,43,44)$. In fact, many intestinal species do not possess a complete synthetic pathway for putrescine production from arginine (45) and therefore, it is possible to suppose the existence of a metabolic pathway spanning multiple bacterial species in the gut $(8,39)$. However, the knowledge about the contribution of gut microbiota to PAs formation is scarce and not sufficient.

\section{THE NEXT-GENERATION PROBIOTIC BACTERIA AND POLYAMINES}

There is an enormous amount of research on probiotics and their beneficial impact on human health. Probiotics are defined by Boirivant and Strober (46) as "live microorganisms that, when administrated in adequate amounts, confer a health benefit on the host." The main sources of probiotics are gut or some fermented foods, such as kefir grains and yogurts, with Lactobacillus spp. and Bifidobacterium spp. being the most used microorganisms. Saccharomyces boulardii, Bacillus spp., E. coli, enterococci, and Weissella spp. are also included. With the development of new methodologies, a new era in probiotic research is started and the new probiotics are referred to next generation probiotics. In fact, there is an increasing interest in the use of gut commensal bacteria as potential probiotics, such as the genera Bacteroides, Clostridium, Bifidobacterium, and Faecalibacterium that predominate in the human gut microbiome (47). The mechanisms of probiotics activity are not clearly understood, even if many studies have been carried out (48). The potential biological effects of probiotics are characterized by an extremely 
diverse range, such as the new functional activities that are currently studied (49).

The species of colonic PAs-producing bacteria are several and different. The PAs concentration in the gut depends on the high or low presence of PAs-producing bacteria and also of PAs-absorbing bacteria. However, the presence of probiotics can increase the concentration of PAs in intestinal lumen as reported after the consumption of yogurt added with probiotics such as Bifidobacterium animalis subsp. lactis LKM512 (34). The consumption of yogurt containing the probiotic strain $B$. animalis subsp. lactis LKM512, increases the PAs concentration in human gut, favoring several positive effects for improving intestinal health, increasing lifespan and quality of life $(33,50$, 51). PAs have been associated with cancer risk and represent a specific marker for neoplastic proliferation. The administration of probiotic Lactobacillus rhamnosus strain GG has been found to affect the synthesis of PAs in gut and the proliferation rates of gastric cell cancer. A relationship between PAs biosynthesis and probiotic action in carcinogenesis and cancer growth was found (52).

The consumption of probiotic strain $B$. animalis subsp. lactis LKM512, colonizes the colon and alters the intestinal microbiota, producing PAs. This alteration in intestinal microbiota favors some bacteria and suppress others, such as Enterobacteriaceae species, and Enterococcus spp. The produced PAs induce maintenance and/or recovery of intestinal barrier function and other beneficial activities such as longevity (53). The activation of PAs biosynthesis is performed by indigenous gut microbiota stimulated by environmental acidification induced by Bifidobacterium. In fact, these microorganisms do not possess enzymes involved in PAs biosynthesis (39).

A study carried out with a cocktail of probiotics, administered for 60 days, enhanced the PAs biosynthesis

\section{REFERENCES}

1. Nicholson JK, Holmes E, Kinross J, Burcelin R, Gibson G, Jia W, et al. Host-gut microbiota metabolic interactions. Science (2012) 336:1262-7. doi: $10.1126 /$ science. 1223813

2. Matsumoto M, Kibe R, Ooga T, Aiba Y, Kurihara S, Sawaki E, et al. Impact of intestinal microbiota on intestinal luminal metabolome. Sci Rep. (2012) 2:233. doi: $10.1038 /$ srep00233

3. Igarashi $\mathrm{K}$, Kashiwagi K. Modulation of cellular function by polyamines. Int $J$ Biochem Cell Biol. (2010) 42:39-51. doi: 10.1016/j.biocel.2009.07.009

4. Murray-Stewart T, Woster PM, Casero Jr RA. Targeting polyamines metabolsim for cancer therapy and prevention. Biochem J. (2016) 437:293753. doi: 10.1042/BCJ20160383

5. Milovic V. Polyamines in the gut lumen: bioavailability and distribution. Eur J Gastr Hepat. (2001) 13:2021-5. doi: 10.1097/00042737-200109000-00004

6. Matsumoto M, Benno Y. The relationship between microbiota and polyamine concentration in the human intestine: a pilot study. Microbiol Immunol. (2007) 51:25-35. doi: 10.1111/j.1348-0421.2007.tb03887.x

7. Kibe R, Kurihara S, Sakai Y, Suzuki H, Ooga T, Sawaki E, et al. Upregulation of colonic luminal polyamines produced by intestinal microbiota delays senescence in mice. Sci Rep. (2014) 4:4548. doi: 10.1038/srep04548

8. Sugiyama Y, Nara M, Sakanaka M, Gotoh A, Kitakata A, Okuda S, et al. Comprehensive analysis of polyamine transport and biosynthesis in the dominant human gut bacteria: Potential presence of novel polyamine metabolism and transport genes. Int J Biochem Cell Biol. (2017) 93:52-61. doi: 10.1016/j.biocel.2017.10.015 in canine inflamed colonic mucosa, regulating PAs levels (54). The administration of mixed probiotic cultures of Lactobacillus spp. strains has been described to induce positive health effects (55). Therefore, the positive effects have been proved in live and dead probiotic preparations [for a review see Adams (56)].

\section{CONCLUSION}

Microbiota-generated metabolites are an essential part of human physiology and are generated through microorganismmicroorganism and host-microorganism interactions, with profound effects on human health and disease. Among the metabolites generated by bacteria in human gut PAs exhibit various beneficial effects, such as increased longevity, recovery of injured mucosa, and favorable effects on cognitive function. However, there is limited knowledge of how microorganisms interact with each other to synthesize metabolites in gut such as PAs. To obtain these tools it will be important to analyse the individual species and strains within these communities including uncultured microorganisms. Future researches on next-generation probiotics and/or mixed cultures of probiotic species should be investigated in order to better understand human health problems in the intestinal tract and find new strategies to face them. PAs modulation by gut microbiota and probiotic consortia could be a good strategy to achieve beneficial effects for human health.

\section{AUTHOR CONTRIBUTIONS}

All authors listed have made a substantial, direct and intellectual contribution to the work, and approved it for publication.

9. Wallace HM, Fraser AV, Hughes A. A perspective of polyamine metabolism. Biochem J (2003) 376:1-14. doi: 10.1042/BJ20031327

10. Nowotarski SL, Woster PM, Casero RA. Polyamines and cancer: implications for chemotherapy and chemoprevention. Expert Rev Mol Med. (2013) 15:e3. doi: $10.1017 /$ erm.2013.3

11. Mariottini P. Spermine metabolism and anticancer therapy. Curr Cancer Drug Targets. (2009) 9:118-30. doi: 10.2174/1568009097875 80935

12. Timmons J, Chang ET, Wang JY, Rao JN. Polyamines and gut mucosal homeostasis. J Gastrointest Dig Syst. (2012) 2(Suppl. 7):001.

13. Miller-Fleming L, Olin-Sandoval V, Campbell K, Ralser M. Remaining mysteries of molecular biology: the role of polyamines in the cell. J Mol Biol. (2016) 427:3389-406. doi: 10.1016/j.jmb.2015.06.020

14. Babbar N, Gerner EW. Targeting polyamines and inflammation for cancer prevention. Recent Res Cancer (2011) 188:49-64. doi: 10.1007/978-3-642-10858-7_4

15. Castelle CJ, Banfield J. Major new microbial group expand diversity and alter our understanding of the tree of life. Cell (2018) 172:1181-97. doi: 10.1016/j.cell.2018.02.016

16. Pugin B, Barcik W, Westermann P, Heider A, Wawrzyniak M, Hellings $\mathrm{P}$, et al. A wide diversity of bacteria from the human gut produces and degrades biogenic amines. Microb Ecol Health Dis. (2017) 28:1353881. doi: 10.1080/16512235.2017.1353881

17. Michael AJ. Biosynthesis of polyamines and polyamine-containing molecules. Biochem J. (2016) 437:2315-29. doi: 10.1042/BCJ 20160185 
18. Michael AJ. Polyamine function in archaea and bacteria. Polyamine J Biol Chem. (2018) 293:18693-701. doi: 10.1074/jbc.TM118.0 05670

19. Hamana K, Matsuzaki S. Polyamines as a chemotaxonomic marker in bacterial systematics. Crit Rev Microbiol. (1992) 18:261-83. doi: 10.3109/10408419209113518

20. Nakamura A, Ooga T, Matsumoto M. Intestinal luminal putrescine is produced by collective biosynthetic pathways of the commensal microbiome. Gut Microbes (2018) 5:1-13. doi: 10.1080/19490976.2018.1494466

21. Wen L, Duffy A. Factors influencing the gut microbiota, inflammation, and type 2 diabetes. J Nutr. (2017) 147:1468S-75S. doi: 10.3945/jn.116.240754

22. Pickard JM, Zeng MY, Caruso R, Núñez G. Gut microbiota: role in pathogen colonization, immune responses, and inflammatory disease. Immunol Rev. (2017) 279:70-89. doi: 10.1111/imr.12567

23. Hillman ET, Lu H, Yao T, Nakatsu CH. Microbial ecology along the gastrointestinal tract. Microbes Environ. (2017) 32:300-13. doi: 10.1264/jsme2.ME17017

24. Hugon P, Lagier JC, Colson P, Bittar F, Raoult D. Repertoire of human gut microbes Microb. Pathogen. (2017) 106:103-12. doi: 10.1016/j.micpath.2016.06.020

25. Barko PC, McMichael MA, Swanson KS, Williams DA. Gastrointestinal microbiome: a Review. J Vet Inter Med. (2018) 32:9-25. doi: 10.1111/jvim.14875

26. Lagier JC, Armougom F, Million M, Hugon P, Pagnier I, Robert C. Microbial culturomics: paradigm shift in the human gut microbiome study. Clin Microbiol Infect. (2012) 18:1185e1193. doi: 10.1111/1469-0691.12023

27. Linsalata M, Orlando A, Russo F. Pharmacological and dietary agents for colorectal cancer chemoprevention: effects on polyamine metabolism. Int J Oncol. (2014) 45:1802-12. doi: 10.3892/ijo.2014.2597

28. Di Martino ML, Campilongo R, Casalino M, Micheli G, Colonna B, Prosseda G. Polyamines: emerging players in bacteria-host interactions. Int $\mathrm{J} \mathrm{Med}$ Microbiol. (2013) 303:484-91. doi: 10.1016/j.ijmm.2013.06.008

29. Igarashi K, Kashiwagi K. Characteristics of cellular polyamine transport in prokaryotes and eukaryotes. Plant Physiol Biochem. (2010) 48:506-12. doi: 10.1016/j.plaphy.2010.01.017

30. Sansonetti PJ. Shigellosis: an old disease in new clothes? PLoS Med. (2006) 3:e354. doi: 10.1371/journal.pmed.0030354

31. Hanfrey CC, Pearson BM, Hazeldine S, Lee J, Gaskin DJ, Woster PM, et al. Alternative spermidine biosynthetic route is critical for growth of Campylobacter jejuni and is the dominant polyamine pathway in human gut microbiota. J Biol Chem. (2011) 286:43301-12. doi: 10.1074/jbc.M111.307835

32. Mortensen PB, Clausen MR. Short-chain fatty acids in the human colon: relation to gastrointestinal health and disease. Scand J Gastr. (1996) 216:13248. doi: 10.3109/00365529609094568

33. Matsumoto M, Aranami A, Ishige A, Watanabe K, Benno Y. LKM512 yogurt consumption improves the intestinal environment and induces the Th1- type cytokine in adult patients with intractable atopic dermatitis. Clin Exp Allergy (2007) 37:358-70. doi: 10.1111/j.1365-2222.2007.02642.x

34. Matsumoto M, Benno Y. Consumption of Bifidobacterium lactis LKM512 yogurt reduces gut mutagenicity by increasing gut polyamine contents in healthy adult subjects. Mutat Res. (2004) 568:147-53. doi: 10.1016/j.mrfmmm.2004.07.016

35. Noack J, Dongowski G, Hartmann L, Blaut M. The human gut bacteria Bacteroides thetaiotaomicron and Fusobacterium varium produce putrescine and spermidine in cecum of pectin-fed gnotobiotic rats. J Nutr. (2000) 130:1225-31. doi: 10.1093/jn/130.5.1225

36. Noack JN, Kleessen B, Proll J, Dongowski G, Blaut M. Dietary guar gum and pectin stimulate intestinal microbial polyamine synthesis in rats. J Nutr. (1998) 128:1385-91. doi: 10.1093/jn/128.8.1385

37. Sakanaka M, Sugiyama Y, Kitakata A, Katayama T, Kurihara S. Carboxyspermidine decarboxylase of the prominent intestinal microbiota species Bacteroides thetaiotaomicron is required for spermidine biosynthesis and contributes to normal growth. Amino Acids. (2016) 48:2443-51. doi: 10.1007/s00726-016-2233-0

38. Burrell M, Hanfrey CC, Murray EJ, Stanley-Wall NR, Michael AJ. Evolution and multiplicity of arginine decarboxylases in polyamine biosynthesis and essential role in Bacillus subtilis biofilm formation. J Biol Chem. (2010) 285:39224-38. doi: 10.1074/jbc.M110.163154

39. Kitada Y, Muramatsu K, Toju H, Kibe R, Benno Y, Kurihara S, et al. Bioactive polyamine production by a novel hybrid system comprising multiple indigenous gut bacterial strategies. Sci. Adv. (2018) 4:eaat0062. doi: $10.1126 /$ sciadv.aat0062

40. Richard H, Foster JW. Escherichia coli glutamate- and argininedependent acid resistance systems increase internal $\mathrm{pH}$ and reverse transmembrane potential. J. Bacteriol. (2004) 186:6032-41. doi: 10.1128/JB.186.18.6032-6041.2004

41. Gong S, Richard H, Foster JW. YjdE (AdiC) is the arginine:agmatine antiporter essential for arginine-dependent acid resistance in Escherichia coli. J Bacteriol. (2003) 185:4402-9. doi: 10.1128/JB.185.15.4402-4409.2003

42. Suárez C, Espariz M, Blancato VS, Magni C. Expression of the agmatine deiminase pathway in Enterococcus faecalis is activated by the AguR regulator and repressed by CcpA and PTSMan systems. PLos ONE (2013) 8:e76170. doi: 10.1371/journal.pone.0076170

43. Atiya Ali M, Poortvliet E, Strömberg R, Yngve A. Polyamines in foods: development of a food database. Food Nutr Res. (2011) 55:5572. doi: 10.3402/fnr.v55i0.5572

44. Bardocz S, Grant G, Brown DS, Ralph A, Pusztai A. Polyamines in food-implications for growth and health J. Nutr Biochem. (1993) 4:66-71. doi: 10.1016/0955-2863(93)90001-D

45. Qin J, Li R, Raes J, Arumugam M, Burgdorf KS, Manichanh C, et al. A human gut microbial gene catalogue established by metagenomic sequencing. Nature (2010) 464:59-65. doi: 10.1038/nature08821

46. Boirivant M, Strober W. The mechanism of action of probiotics. Curr Opin Gastroenterol. (2007) 23:679-92. doi: 10.1097/MOG.0b013e3282f0cffc

47. O’Toole PW, Marchesi JR, Hill C. Next-generation probiotics: the spectrum from probiotics to live biotherapeutics. Nat Microbiol. (2017) 2:17057.doi: 10.1038/nmicrobiol.2017.57

48. Daliri EBM, Lee BH. New perspectives on probiotics in health and disease. Food Sci Hum Wellness (2015) 4:56-65. doi: 10.1016/j.fshw.2015.06.002

49. Vandenplas Y, Huys G, Daube G. Probiotics: an update. J Pediatr. (2015) 91:6-21. doi: 10.1016/j.jped.2014.08.005

50. Matsumoto M, Kitada Y, Shimomura Y, Naito Y. Bifidobacterium animalis subsp. lactis LKM512 reduces levels of intestinal trimethylamine produced by intestinal microbiota in healthy volunteers: a doubleblind, placebo-controlled study. J Funct Foods (2017) 36:94-101. doi: $10.1016 /$ j.jff.2017.06.032

51. Matsumoto $\mathrm{M}$, Ohishi $\mathrm{H}$, Benno $\mathrm{Y}$. Impact of LKM512 yogurt on improvement of intestinal environment of the elderly. FEMS Immunol Medical Microbiol. (2001) 31:181-6. doi: 10.1111/j.1574-695X.2001.tb00518.x

52. Linsalata M, Cavallini A, Messa C, Orlando A, Refolo MG, Russo F. Lactobacillus rhamnosus GG influencespolyamine metabolismin HGG27 gastric cancer cell lines: a strategy toward nutritional approach to chemoprevention of gastric cancer. Curr Pharm Des. (2010) 16:847-53. doi: 10.2174/138161210790883598

53. Matsumoto M, Kurihara S, Kibe R, Ashida H, Benno Y. Longevity in mice is promoted by probiotic-induced suppression of colonic senescence dependent on upregulation of gut bacterial polyamine production. PLos ONE (2011) 6:e23652. doi: 10.1371/journal.pone.0023652

54. Rossi G, Cerquetella M, Scarpona S, Pengo G, Fettucciari K, Bassotti G, et al. Effects of probiotic bacteria on mucosal polyamines levels in dogs with IBD and colonic polyps: a preliminary study. Benef Microbes (2018) 9:247-55. doi: 10.3920/BM2017.0024

55. Orlando A, Messa C, Linsalata M, Cavallini A, Russo F. Effects of Lactobacillus rhamnosus GG on proliferation and polyamine metabolism in HGC-27 human gastric and DLD-1 colonic cancer cell lines. Immunopharmacol Immunotoxicol. (2009) 31:108-16. doi: 10.1080/08923970802443631

56. Adams CA. The probiotic paradox: live and dead cells are biological response modifiers. Nutr Res Rev. (2010) 23:37-46. doi: 10.1017/S0954422 410000090

Conflict of Interest Statement: The authors declare that the research was conducted in the absence of any commercial or financial relationships that could be construed as a potential conflict of interest.

Copyright (C) 2019 Tofalo, Cocchi and Suzzi. This is an open-access article distributed under the terms of the Creative Commons Attribution License (CC BY). The use, distribution or reproduction in other forums is permitted, provided the original author(s) and the copyright owner(s) are credited and that the original publication in this journal is cited, in accordance with accepted academic practice. No use, distribution or reproduction is permitted which does not comply with these terms. 